\title{
Cardiovascular effects of alfaxalone on hemodynamic function in pigs
}

This article was published in the following Dove Press journal:

Open Access Animal Physiology

14 August 2013

Number of times this article has been viewed

\author{
Nadine Pfeiffer ${ }^{1}$ \\ Johanna Ebner' \\ Anne-Kathrin von Thaden' \\ Tibor Schuster ${ }^{2}$ \\ Wolf Erhardt ${ }^{3}$ \\ Christine Baumgartner \\ 'Center of Preclinical Research, \\ Klinikum rechts der Isar, Technical \\ University of Munich, Munich, \\ Germany; ${ }^{2}$ Institute for Medical \\ Statistics and Epidemiology, Klinikum \\ rechts der Isar, Technical University \\ of Munich, Munich, Germany; \\ ${ }^{3}$ Institute for Experimental Oncology \\ and Therapeutic Research, Klinikum \\ rechts der Isar, Technical University \\ of Munich, Munich, Germany
}

Background: The purpose of this study was to investigate the short-term cardiovascular effects of intravenous (IV) alfaxalone bolus injections in healthy pigs.

Materials and methods: Each pig was sedated with ketamine, azaperone, and atropine intramuscularly, mixed in one syringe. Anesthesia was induced and maintained intravenously with alfaxalone. Then, three defined test bolus injections of alfaxalone were given intravenously. Vascular ultrasonography and pulse-induced contour cardiac output thermodilution monitoring were used to evaluate the direct cardiovascular effects after bolus injection. The following vascular and hemodynamic variables were recorded at the right common carotid artery (CCA) for 10 minutes after each alfaxalone injection by ultrasonography, pulse oximetry, and capnometry: peak systolic, minimum diastolic, end-diastolic, and time-averaged blood flow velocities; average volumetric flow; resistance index and vessel diameter; heart rate (HR); arterial oxygen saturation; and end-tidal carbon dioxide $\left(\mathrm{PETCO}_{2}\right)$. In addition, pulse contour-derived cardiac output recorded cardiac output, contractility, and volumetric variables for preload and afterload, as well as extravascular lung water, blood temperature, arterial blood pressure, and central venous pressure.

Results: Alfaxalone bolus injections caused an initial short-lasting ( 0.25 minutes) decrease in diameter at the CCA after each bolus. During this very acute reaction, peak systolic blood flow velocity, thermodilutional cardiac output, and HR increased significantly. Average volumetric flow decreased, but not significantly. Mean arterial blood pressure decreased significantly at a time point of 0.25 minutes. Stroke volume, end-diastolic blood flow velocity, and resistance index did not change significantly. No cumulative effect was seen when three bolus injections of alfaxalone were given.

Conclusion: Alfaxalone bolus injections produced two-phased short-lasting vascular (vasoconstriction at the CCA, vasodilation in the peripheral vessels) and hemodynamic changes (HR, mean arterial pressure), which were easily compensated by young healthy pigs. Because of those very short changes, alfaxalone is a safe hypnotic agent in healthy pigs; however, it may be used carefully in patients with ventricular dysfunction.

Keywords: anesthetics, cardiovascular, alfaxalone, ultrasonography, pigs

\section{Introduction}

Originally, alfaxalone was coformulated with the steroid analgesic alfadolone using Cremophor $^{\circledR}$ EL (BASF SE, Ludwigshafen, Germany) as an excipient (Althesin ${ }^{\circledR}$, GlaxoSmithKline, Brentford, UK; Saffan ${ }^{\circledR}$, Merck Animal Health, Summit, NJ, USA). The side effects of this formulation are well known: Cremophor ${ }^{\circledR}$ EL (BASF SE) induces histamine release in cats, dogs, and humans, which leads to edema of the pinnae and paws, hyperemia, or anaphylactoid reactions. ${ }^{1-3}$ Alfaxan ${ }^{\circledR}$ (Jurox Pty Limited, Rutherford, 
NSW, Australia) is formulated without alfadolone and is free of Cremophor ${ }^{\circledR}$ EL (BASF SE). In addition, 2-hydroxypropylbeta cyclodextrin is the excipient in this new formulation, which does not cause histamine liberation. It is a large oligosaccharide molecule that embraces alfaxalone and has a hydrophilic exterior, which consequently makes the complex soluble in water. ${ }^{4}$ Alfaxalone (Alfaxan ${ }^{\circledR}$, Jurox Pty Limited) is already licensed in Australia, New Zealand, South Africa, the United Kingdom, and Thailand.

Since 2008, alfaxalone has also been licensed in Germany for use in cats and dogs for the induction and maintenance of anesthesia. Alfaxalone ( $3 \alpha$-hydroxy- $5 \alpha$-pregnane11,20 -dione) is a steroid hypnotic agent with a short duration of action because of its fast redistribution and metabolism; ${ }^{5}$ therefore, it is well controllable. Anesthesia can be induced with and without premedication and can be maintained with alfaxalone, producing good hypnosis and good muscle relaxation. ${ }^{6-12}$ The muscle tonus is reduced; therefore, intubation of the patient is easy. ${ }^{8}$ In studies conducted with dogs, apnea was more likely to occur when induction of anesthesia was conducted with propofol than with alfaxalone. ${ }^{13}$

To the best of our knowledge, sparse information exists about the use of alfaxalone in pigs. ${ }^{10}$ Its analgesic properties are very poor, so it is only classified as a hypnotic agent. Consequently, surgical procedures should only be carried out when alfaxalone is combined with an analgesic. The cardiovascular effects of alfaxalone have been described to produce a decrease in vascular resistance resulting in hypotension, but it has no negative inotropic effect on the heart. ${ }^{5}$ However, either for induction or to deepen anesthesia, alfaxalone is given as a bolus injection.

The purpose of this study was to evaluate the direct short-term effects of alfaxalone bolus injections on the cardiovascular system using pulse-induced contour cardiac output (PiCCO) and vascular ultrasound monitoring.

The PiCCO, an advanced method that is often used in human intensive care medicine, was chosen in our study to verify the short-term cardiovascular effects (especially regarding the cardiac output $[\mathrm{CO}]$ and volumetric variables for preload and afterload) of alfaxalone.

Two methods are combined in the PiCCO technique: (1) a discontinuous method, which is based on transcardiopulmonal thermodilution; and (2) a continuous method, which is based on pulse contour analysis.

With the PiCCO technique, the following hemodynamic parameters can be measured: heart rate (HR), stroke volume (SV), CO, SV variation (SVV), systemic vascular resistance index (SVRI), global end-diastolic volume index (GEDI), extravascular lung water index, maximum velocity of pressure rise in the aorta (dPmax), global extraction fraction (GEF), and cardiac function index (CFI).

Several studies have been conducted in pigs comparing transcardiopulmonal thermodilution values (derived from the PiCCO technique) with the pulmonary arterial thermodilution technique, which has been the gold standard in measuring hemodynamic variables since its invention in 1970, resulting in comparable data by both techniques. ${ }^{14-17}$

Alfaxalone has already been investigated in dogs, cats, rabbits, and sheep, ${ }^{5-9,18-20}$ but to the best of our knowledge, only one study has been conducted in pigs. ${ }^{10}$ In 2003, Keates ${ }^{10}$ used alfaxalone as an induction agent after premedication with azaperone and evaluated the necessary dose rate to produce satisfactory conditions for intubation. The objective of our study was to evaluate the direct, short-term vascular and hemodynamic changes of three intravenous (IV) bolus injections of alfaxalone during alfaxalone anesthesia in pigs.

\section{Materials and methods Animals}

Six 3-month-old female landrace pigs weighing $34.5 \pm 1.9 \mathrm{~kg}$ (mean \pm standard deviation [SD]) were used in this study. The study protocol was authorized by the local animal care committee and was carried out in accordance with the German Animal Welfare Act (Deutsches Tierschutzgesetz).

The pigs were obtained from an experimental breeding colony (Versuchsstation Thalhausen, Germany) and were group-housed ( $3 \mathrm{~m}^{2}$ for two animals) under conventional hygienic conditions. The air temperature was maintained at $19^{\circ} \mathrm{C} \pm 2{ }^{\circ} \mathrm{C}$, and relative humidity was $50 \%-60 \%$. The pigs were provided with commercial pelleted forage (deuka Kornmast 130 gekörnt, Alleinfuttermittel für Mastschweine, Deutsche Tiernahrung Cremer GmbH and Co KG, Regensburg, Germany) twice daily and received water ad libitum. All animals were housed at least 7 days before the day of the experiment to adapt to the new environment and were kept on a 12-hour light/dark cycle. For enrichment, plastic balls were provided. At 12 hours before induction of anesthesia, the pigs were starved but still had free access to water.

\section{Anesthesia}

On the day of the experiment, each pig was weighed and examined adspectorically. Experiments were conducted between 8 am and $12 \mathrm{pm}$. Sedation was induced by $10 \mathrm{mg} / \mathrm{kg}$ of ketamine (Narketan $10^{\circledR}$; Vétoquinol, Ravensburg, Germany), $2 \mathrm{mg} / \mathrm{kg}$ of azaperone (Stresnil ${ }^{\circledR}$; Janssen Animal Health, 
Neuss, Germany), and $0.029 \mathrm{mg} / \mathrm{kg}$ of atropine (Atropin ${ }^{\circledR}$; B Braun Medical Inc, Melsungen, Germany) intramuscularly in one syringe. A $0.9 \times 25 \mathrm{~mm}$ catheter (Vasofix; B Braun Medical Inc) was placed into the right and left lateral auricular veins. Anesthesia was induced intravenously (IV) using $0.7 \mathrm{mg} / \mathrm{kg}$ of alfaxalone (Alfaxan ${ }^{\circledR}$; Vétoquinol) after the animals became recumbent. The trachea was intubated (7-7.5 mm internal diameter of tube) after loss of the swallowing reflex. Then, a single bolus of $40 \mathrm{mg} / \mathrm{kg}$ metamizole (Novaminsulfon-ratiopharm $2.5^{\circledR}$; Ratiopharm GmbH, Ulm, Germany) was given intravenously for preemptive analgesia. Each pig was mechanically ventilated (Cicero; Draeger Medizintechnik, Luebeck, Germany) with 100\% oxygen at $12-17$ breaths per minute and a peak ventilation pressure of $8-10 \mathrm{~cm}$ of $\mathrm{H}_{2} \mathrm{O}$. A pulse oximeter (8600V; Nonin Medical, Inc, Plymouth, MN, USA) was used to observe HR and peripheral arterial oxygen saturation $\left(\mathrm{SpO}_{2}\right)$. End-tidal carbon dioxide $\left(\mathrm{PETCO}_{2}\right)$ and rectal body temperature were controlled; in addition, the central venous pressure was measured with a patient monitor (Datex Ohmeda S/5 Type F-CM1.00 pressure transducers, Hellige Type 4-327-I; Datex Ohmeda, Helsinki, Finland).

The pigs were placed in the dorsal recumbent position to allow ultrasonographic measurements of the right common carotid artery (CCA). A continuous IV infusion of alfaxalone $(13.2 \pm 0.7 \mathrm{mg} / \mathrm{kg} /$ hour$)$ was given to proceed with anesthesia. With this dose, a stable and light plane of anesthesia was maintained, producing good muscle relaxation and hypnosis with stable cardiovascular variables (HR, mean arterial blood pressure [MAP], $\mathrm{SpO}_{2}, \mathrm{PETCO}_{2}$, and ultrasonographic parameter).

Saline was infused at a maintenance rate of $10 \mathrm{~mL} / \mathrm{kg} / \mathrm{hour}$. All additional fluids for PiCCO measurements were subtracted from the infusion rate not to exceed the maintenance rate $(10 \mathrm{~mL} / \mathrm{kg} /$ hour $)$.

\section{Experimental protocol}

Adequate analgesia during the insertion of vascular catheters into the left external jugular vein (tube/X-ray line $50 \mathrm{~cm}$ CH08; Unomedical a/s, Birkerød, Denmark) and into a superficial branch of the left iliac artery $\left(\mathrm{PiCCO}^{\circledR}\right.$ brachial artery catheter $16 \mathrm{~cm}$ 4F; PVPK2014 L16-46 N, PULSION Medical Systems AG, Munich, Germany) was achieved by $0.007 \mathrm{mg} / \mathrm{kg} /$ hour of remifentanil constant rate infusion (CRI) (Ultiva $^{\circledR} 5 \mathrm{mg}$; GlaxoSmithKline, Munich, Germany). After the invasive procedures were completed, remifentanyl CRI was stopped. To ensure that only the alfaxalone bolus injections were affecting the cardiovascular system, we waited for a washing-out period of at least 10 minutes ( $15 \pm 7.16$ minutes $)$ for remifentanyl.

Baseline measurements were determined only after a stable anesthetic plane was apparent (ie, values for HR, MAP, $\mathrm{PETCO}_{2}$, and ultrasonographic variables did not differ from respective baseline values of more than 5\%).

Every 25 minutes, a bolus injection of alfaxalone IV $(1.5 \mathrm{mg} / \mathrm{kg})$ was administered. In total, three bolus injections were given. Each bolus was injected during a period of 10 seconds. The end of each alfaxalone injection was defined as Time 0 . After each of the three alfaxalone bolus injections, changes in vascular and hemodynamic variables were recorded for 10 minutes using ultrasonography and PiCCO thermodilution monitoring (recorded time points: 15 seconds and minutes 1-10).

During ultrasonographic ascertainment of the CCA, the vessel images and velocity spectra were recorded for determination of vessel diameter, peak systolic blood flow velocity (psBFV), minimum diastolic blood flow velocity (mdBFV), end-diastolic blood flow velocity (edBFV), and time-averaged blood flow velocity (VF).

The resistance index (RI) of the blood vessels, which is derived from the psBFV and edBFV, reflects the vascular resistance distal to the point of Doppler sampling. It was defined according to the following calculation:

$$
(\mathrm{psBFV}-\mathrm{edBFV}) / \mathrm{psBFV} \text {, }
$$

where psBFV is the peak systolic BFV and edBFV is the end-diastolic BFV. Mean volumetric flow in the right CCA was calculated using the formula,

$$
\mathrm{V}_{\text {mean }} \times \pi \times \mathrm{r}^{2}
$$

where $\mathrm{V}_{\text {mean }}$ is the mean velocity and $\mathrm{r}$ is the vessel radius.

\section{$\mathrm{PiCCO}$ thermodilution measurements}

As mentioned above, the PiCCO technique uses two methods to evaluate hemodynamic parameters: (1) a discontinuous method, which is based on transcardiopulmonal thermodilution; and (2) the continuous method, which is based on pulse contour analysis.

Transcardiopulmonal thermodilution uses cold saline as an indicator. In our study, $10 \mathrm{~mL}$ of cold $\left(\sim 8^{\circ} \mathrm{C}\right)$ sodium chloride was injected at 1 minute, 2 minutes, 4 minutes, 6 minutes, 8 minutes, and 10 minutes after a test bolus application of alfaxalone into the left jugular vein via the PiCCO injection pin. Then, the cold sodium chloride was distributed 
via blood flow to the right side of the heart, into the lungs, and to the left side of the heart, and it passed the aorta and the femoral and iliac arteries to arrive at the thermistor probe placed in the superficial branch of the left iliac artery. PiCCO calculated CO, CFI, GEDI, extravascular lung water index, and the GEF using the thermodilution curve.

With the second method - continuous pulse contour analysis - the following parameters were recorded at 15 seconds and minutes 1-10 after alfaxalone bolus injection: HR, arterial blood pressure (systolic and diastolic blood pressures and MAP), CO, SVRI, SVV, parameter for left ventricular contractility (dPmax), and blood temperature.

Consequently, two different methods were used to measure $\mathrm{CO}$ with the PiCCO technique included in our study: thermodilution and pulse contour analysis.

$\mathrm{SV}$ and HR affect CO. Furthermore, SV is dependent on three different parameters: contractility, preload, and afterload. The contractility of the heart is defined via the parameters dPmax, GEF, and CFI. The dPmax describes the contractility of the left ventricle. The equation,

$$
4 \times \mathrm{SV} / \mathrm{GEDV}
$$

calculates the GEF. The CFI is calculated by the equation,

\section{$\mathrm{CO} / \mathrm{GEDV}$,}

and reflects the fraction of preload volume, which is pumped by the heart in 1 minute.

Using the PiCCO technique, the SVV determines whether an increase in preload (for example, because of infusion) in a controlled, ventilated patient leads to an increase in CO. The GEDI is another important parameter that defines the preload of the heart using the PiCCO technique. GEDI is the global end-diastolic volume (GEDV) referred to body surface, indicating the effect of external infusion on the circulation. The SVRI is a parameter that defines the afterload. During PiCCO measurements, $\mathrm{HR}, \mathrm{SpO}_{2}, \mathrm{PETCO}_{2}$, and rectal body temperature were recorded simultaneously.

\section{Ultrasonography of the right CCA}

Vascular imaging of the right CCA was conducted with an ultrasonographic system (Vivid 7; GE Vingmed, Horten, Norway) with a $10 \mathrm{MHz}$ linear transducer (FLA 1-MHz 1A; GE Vingmed). The probe was placed on the right side of the ventral cervical region and was adjusted until distinct parallel vessel walls were visible on the ultrasonic image of the CCA. A clamp fixed the probe in that position. Adjustment of instrument settings was done to delineate the vessel walls from surrounding tissues.

Afterward, the Doppler sample volume was placed centrally in the vessel. The sample volume cursor was adjusted to align with the vessel walls and blood flow. An angle of $45^{\circ}$ to $60^{\circ}$ between the vessel and the ultrasound beam was measured in the ultrasound image and was adhered to in every experiment. This angle was used to correct velocity calculations. Once the sample volume was positioned correctly, ultrasonic measurements were performed. Doppler evaluations were implemented in pulsed-wave mode. Velocity spectra were surveyed using the visual and audible signals and then were saved for psBFV, mdBFV, edBFV, and VF. The RI, pulsatility index, and mean volumetric flow were figured up from these BFV data. Moreover, the luminal diameter was calculated by cursor adjustment using two-dimensional images of the vessel wall that were recorded and stored previously. Averaged volumetric flow in the right CCA was calculated using the equation,

$$
\mathrm{V}_{\text {mean }} \times \pi \times \mathrm{r}^{2}
$$

where $r$ is the vessel radius and $V_{\text {mean }}$ is the mean velocity. ${ }^{21}$

\section{Statistical evaluation}

Descriptive statistics (mean $\pm \mathrm{SD}$ ) were reported for all data. Statistical comparisons were made for an exploratory data analysis; thus, no correction of a type 1 error was considered. All statistical tests were conducted two-sided, and a $P$-value of less than 0.05 was considered to indicate statistical significance.

To evaluate the overall time trend of interesting response variables, linear mixed regression models with monotonous (linear), transient (quadratic), and cubic time effects were fitted to the data. The linear mixed regression modeling approach properly reflects the structure of repeated data and accounts for correlation among measurements within the same subject. A first-order autoregressive correlation structure, as well as random effects for each pig, was considered in the regression analysis. Effects of time were first specified by graphical assessment and then were verified by stepwise model derivation. When a specific effect of time was detected in the global trend analysis, a post hoc Student's $t$-test for paired samples was used to assess differences between each 
time point during the 10-minute examination period and the baseline value. Furthermore, a Friedman's test followed by paired Student's $t$-tests was used to separately compare related samples given by equivalent time points after the first, second, and third bolus for the variables HR, MAP, SVRI, thermodilutional CO (TDCO) and pulse contour CO (PCCO), and SV.

All statistical analyses were conducted with the commercially available software SPSS ${ }^{\circledR}$ (Statistical Package for the Social Sciences, version 19; IBM Corporation, Armonk, NY, USA).

\section{Results}

\section{Ultrasonography of the right CCA}

The data (mean \pm SD) of the measurements at the CCA after the first, second, and third alfaxalone test bolus injections are shown in Tables 1-3. A significant decrease in the diameter of the CCA was obvious after every bolus injection at a time point of 15 seconds. The luminal diameter decreased by $3.2 \%$ after the first bolus, $3.9 \%$ after the second bolus, and $4.6 \%$ after the third bolus compared with baseline values, and the diameter increased again to baseline values at a time point of 1 minute. In this acute phase, psBFV increased significantly after every bolus injection, whereas the other variables did not change significantly. At a time point of 1 minute, psBFV decreased again, but this was only significant after the second alfaxalone bolus.

After this acute, transient phase, several significant changes at different time points in averaged volumetric flow were recorded. The averaged volumetric flow increased at minute 4 and minute 6 after the first bolus injection.

When all data were compared at equivalent time points after the first, second, and third bolus injections, differences in diameter and RI were visible (Table 1). Between the first and second bolus injections, significant differences were seen at minute 9 in RI. At minute 6 and minute 9 , the vessel diameter was significantly different between the first and third bolus injections.

\section{Clinical hemodynamic parameters recorded with $\mathrm{PiCCO}$}

Tables 4-6 show data recorded after injection of the first, second, and third alfaxalone bolus injections. HR increased transiently at a time point of 15 seconds after every bolus injection, but this increase was only significant after the third bolus injection. MAP significantly and transiently decreased

Table I Ultrasonographic evaluation of the right common carotid artery after the first alfaxalone bolus injection (mean \pm SD)

\begin{tabular}{|c|c|c|c|c|c|}
\hline $\begin{array}{l}\text { Time } \\
\text { (minutes) }\end{array}$ & $D(\mathrm{~mm})$ & $\begin{array}{l}\text { psBFV } \\
\text { (cm/second) }\end{array}$ & $\begin{array}{l}\text { edBFV } \\
\text { (cm/second) }\end{array}$ & $\mathbf{R I}(-)$ & $\begin{array}{l}\text { VFave } \\
\text { (mL/second) }\end{array}$ \\
\hline \multicolumn{6}{|l|}{ LMM } \\
\hline Intercept & 4.323 & 116.765 & 19.796 & 0.821 & 5.642 \\
\hline Time $\times$ coef $_{1}$ & 0.045 & -18.065 & 0.082 & -0.045 & 0.370 \\
\hline Time $^{2} \times$ coef $_{2}$ & $-0.003^{*}$ & 3.492 & $* *$ & 0.010 & $-0.032^{*}$ \\
\hline Time $^{3} \times$ coef $_{3}$ & $* * *$ & $-0.187^{*}$ & $* * *$ & $-0.00 I^{*}$ & $* * *$ \\
\hline Baseline & $4.38 \pm 0.20$ & $103.20 \pm 51.73$ & $19.94 \pm 10.28$ & $0.80 \pm 0.11$ & $6.08 \pm 1.98$ \\
\hline 0.25 & $4.24 \pm 0.26 *$ & $|44.4| \pm 50.59 *$ & $18.86 \pm 7.82$ & $0.86 \pm 0.07$ & $4.68 \pm 2.58$ \\
\hline I & $4.30 \pm 0.17$ & $102.25 \pm 54.34$ & $\mid 8.33 \pm 8.81$ & $0.80 \pm 0.11$ & $6.08 \pm 2.35$ \\
\hline 2 & $4.40 \pm 0.23$ & $101.74 \pm 50.75$ & $20.50 \pm 9.57$ & $0.78 \pm 0.10$ & $6.39 \pm 1.81$ \\
\hline 3 & $4.42 \pm 0.16$ & $100.87 \pm 47.24$ & $22.34 \pm 6.77$ & $0.75 \pm 0.10$ & $6.53 \pm 1.64$ \\
\hline 4 & $4.43 \pm 0.19$ & $97.73 \pm 52.33$ & $20.88 \pm 7.91$ & $0.77 \pm 0.11$ & $6.50 \pm 1.86 *$ \\
\hline 5 & $4.40 \pm 0.11$ & $|03.72 \pm 51.7|$ & $20.77 \pm 7.43$ & $0.77 \pm 0.11$ & $6.17 \pm 1.58$ \\
\hline 6 & $4.47 \pm 0.18^{b}$ & $103.33 \pm 43.99$ & $20.44 \pm 7.45$ & $0.78 \pm 0.10$ & $6.68 \pm 1.62 *$ \\
\hline 7 & $4.45 \pm 0.20$ & $105.09 \pm 46.75$ & $20.26 \pm 7.52$ & $0.78 \pm 0.10$ & $6.49 \pm 1.60$ \\
\hline 8 & $4.46 \pm 0.16$ & $104.77 \pm 47.74$ & $18.77 \pm 7.94$ & $0.80 \pm 0.11$ & $6.53 \pm 1.65$ \\
\hline 9 & $4.43 \pm 0.17^{b}$ & $103.26 \pm 42.14$ & $19.67 \pm 9.56$ & $0.79 \pm 0.1 \mathrm{I}^{\mathrm{a}}$ & $6.38 \pm 1.18$ \\
\hline 10 & $4.42 \pm 0.18$ & $103.58 \pm 46.18$ & $20.79 \pm 9.08$ & $0.77 \pm 0.13$ & $6.12 \pm 1.27$ \\
\hline
\end{tabular}

Notes: *Significant differences from baseline $(P<0.05)$; **no quadratic time trend obvious by data assessment; $* * *$ no cubic time trend obvious by data assessment. LMM with

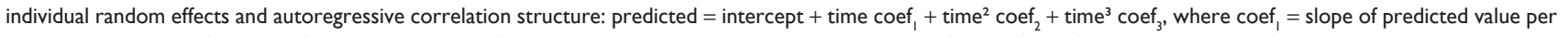
I-minute increment of time; coef $_{2}=$ additive change of predicted value in dependence on squared time $\left(\right.$ minute $\left.^{2}\right)$; coef $_{3}=$ additive change of predicted value in dependence

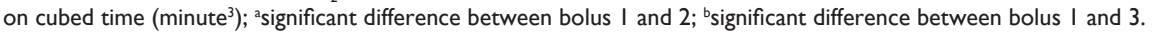

Abbreviations: SD, standard deviation; D, luminal diameter; psBFV, peak systolic blood flow velocity; edBFV, end-diastolic blood flow velocity; RI, resistance index; VFave, average volumetric flow; LMM, linear mixed model. 
Table 2 Ultrasonographic evaluation of the right common carotid artery after the second alfaxalone bolus injection (mean \pm SD)

\begin{tabular}{|c|c|c|c|c|c|}
\hline $\begin{array}{l}\text { Time } \\
\text { (minutes) }\end{array}$ & $D(\mathrm{~mm})$ & $\begin{array}{l}\text { psBFV } \\
\text { (cm/second) }\end{array}$ & $\begin{array}{l}\text { edBFV } \\
\text { (cm/second) }\end{array}$ & $\mathbf{R I}(-)$ & $\begin{array}{l}\text { VFave } \\
\text { (mL/second) }\end{array}$ \\
\hline \multicolumn{6}{|l|}{ LMM } \\
\hline Intercept & 4.280 & 119.562 & 23.877 & 0.782 & 6.418 \\
\hline Time $\times$ coef $_{1}$ & 0.046 & -17.389 & -0.033 & -0.013 & 0.021 \\
\hline Time $^{2} \times$ coef $_{2}$ & $-0.004^{*}$ & 3.237 & $* *$ & $0.001 *$ & $* *$ \\
\hline Time $^{3} \times$ coef $_{3}$ & $* * *$ & $-0.167^{*}$ & $* * *$ & $* * *$ & $* * *$ \\
\hline Baseline & $4.33 \pm 0.13$ & $105.76 \pm 47.88$ & $24.30 \pm 12.68$ & $0.76 \pm 0.11$ & $6.26 \pm 1.15$ \\
\hline 0.25 & $4.16 \pm 0.16^{*}$ & $148.73 \pm 40.99 *$ & $23.92 \pm 13.87$ & $0.83 \pm 0.10$ & $6.51 \pm 1.59$ \\
\hline I & $4.33 \pm 0.21$ & $101.26 \pm 46.25 *$ & $23.50 \pm 9.92$ & $0.75 \pm 0.13$ & $6.48 \pm 1.56$ \\
\hline 2 & $4.37 \pm 0.18$ & $100.78 \pm 40.77$ & $22.28 \pm 9.23$ & $0.76 \pm 0.12$ & $6.64 \pm 1.66$ \\
\hline 3 & $4.36 \pm 0.16$ & $102.36 \pm 45.64$ & $23.90 \pm 9.30$ & $0.74 \pm 0.13$ & $6.75 \pm 1.03$ \\
\hline 4 & $4.39 \pm 0.18$ & $104.29 \pm 40.86$ & $26.27 \pm 13.25$ & $0.74 \pm 0.12$ & $6.96 \pm 1.37$ \\
\hline 5 & $4.40 \pm 0.23$ & $102.74 \pm 37.76$ & $23.77 \pm 9.60$ & $0.75 \pm 0.12$ & $6.68 \pm 1.15$ \\
\hline 6 & $4.37 \pm 0.18$ & $104.12 \pm 39.35$ & $22.66 \pm 7.97$ & $0.77 \pm 0.11$ & $6.73 \pm 1.17$ \\
\hline 7 & $4.35 \pm 0.14$ & $86.69 \pm 4.03$ & $22.58 \pm 9.04$ & $0.77 \pm 0.10$ & $6.50 \pm 1.14$ \\
\hline 8 & $4.38 \pm 0.15$ & $104.93 \pm 37.86$ & $21.94 \pm 9.87$ & $0.70 \pm 0.16$ & $6.86 \pm 1.15$ \\
\hline 9 & $4.37 \pm 0.19$ & $100.94 \pm 31.40$ & $23.63 \pm 9.51$ & $0.76 \pm 0.11$ & $6.83 \pm 1.14$ \\
\hline 10 & $4.34 \pm 0.18$ & $104.93 \pm 35.25$ & $24.11 \pm 9.52$ & $0.76 \pm 0.12$ & $6.58 \pm 0.73$ \\
\hline
\end{tabular}

Notes: *Significant differences from baseline $(P<0.05)$. LMM with individual random effects and autoregressive correlation structure: predicted $=$ intercept + time coef $I+$ time $^{2}$ coef $_{2}+$ time $^{3}$ coef $_{3}$, where coef $f_{1}=$ slope of predicted value per I-minute increment of time; coef $_{2}=$ additive change of predicted value in dependence on squared time $\left.(\text { minute })^{2}\right)$ coef $_{3}=$ additive change of predicted value in dependence on cubed time $\left(m i n u t e^{3}\right) ; * *$ no quadratic time trend obvious by data assessment; ***kno cubic time trend obvious by data assessment.

Abbreviations: SD, standard deviation; D, luminal diameter; psBFV, peak systolic blood flow velocity; edBFV, end-diastolic blood flow velocity; RI, resistance index; VFave, average volumetric flow; LMM, linear mixed model.

at 15 seconds after the first and second alfaxalone injections. After this rapid decrease, MAP immediately started to increase again (not significantly) to reach baseline values at a time point of 1 minute and increased further (significantly after the first and second bolus injections). Furthermore, SVRI decreased significantly after each bolus injection at a time point of 0.25 minutes, then increased continuously after each bolus injection (significantly after the first bolus injection). TDCO significantly and transiently increased after the second bolus injection at a time point of 0.25 minutes; after the first

Table 3 Ultrasonographic evaluation of the right common carotid artery after the third alfaxalone bolus injection (mean \pm SD)

\begin{tabular}{|c|c|c|c|c|c|}
\hline $\begin{array}{l}\text { Time } \\
\text { (minutes) }\end{array}$ & $D(\mathrm{~mm})$ & $\begin{array}{l}\text { psBFV } \\
\text { (cm/second) }\end{array}$ & $\begin{array}{l}\text { edBFV } \\
\text { (cm/second) }\end{array}$ & $\mathbf{R I}(-)$ & $\begin{array}{l}\text { VFave } \\
\text { (mL/second) }\end{array}$ \\
\hline \multicolumn{6}{|l|}{ LMM } \\
\hline Intercept & 4.293 & 107.983 & 21.492 & 0.769 & 6.229 \\
\hline Time $\times$ coef $_{1}$ & 0.027 & -15.076 & 1.220 & -0.021 & -0.001 \\
\hline Time $^{2} \times$ coef $_{2}$ & $-0.002 *$ & 2.790 & -0.126 & $0.002 *$ & $* *$ \\
\hline $\mathrm{Time}^{3} \times \mathrm{coef}_{3}^{2}$ & $* * *$ & $-0.145^{*}$ & $-0.00 I^{*}$ & $* * *$ & $* * *$ \\
\hline Baseline & $4.36 \pm 0.20$ & $97.79 \pm 41.61$ & $21.28 \pm 8.95$ & $0.74 \pm 0.13$ & $6.44 \pm 1.04$ \\
\hline 0.25 & $4.16 \pm 0.14^{*}$ & $129.22 \pm 50.46 *$ & $18.85 \pm 7.95$ & $0.83 \pm 0.12$ & $5.62 \pm 1.31$ \\
\hline 1 & $4.31 \pm 0.15$ & $94.47 \pm 39.42$ & $23.40 \pm 8.77$ & $0.74 \pm 0.13$ & $6.07 \pm 1.04$ \\
\hline 2 & $4.30 \pm 0.17$ & $94.14 \pm 36.32$ & $25.65 \pm 12.50$ & $0.70 \pm 0.14$ & $6.13 \pm 0.99$ \\
\hline 3 & $4.33 \pm 0.15$ & $92.26 \pm 40.56$ & $22.61 \pm 8.50$ & $0.73 \pm 0.14$ & $6.14 \pm 1.01$ \\
\hline 4 & $4.38 \pm 0.14$ & $95.50 \pm 32.46$ & $22.37 \pm 8.40$ & $0.75 \pm 0.13$ & $6.47 \pm 1.01$ \\
\hline 5 & $4.39 \pm 0.19$ & $94.58 \pm 33.90$ & $23.51 \pm 8.04$ & $0.73 \pm 0.13$ & $6.59 \pm 1.16$ \\
\hline 6 & $4.33 \pm 0.14$ & $92.98 \pm 28.77$ & $22.30 \pm 8.29$ & $0.74 \pm 0.13$ & $6.25 \pm 0.93$ \\
\hline 7 & $4.41 \pm 0.16$ & $104.38 \pm 33.10$ & $29.00 \pm 11.47$ & $0.71 \pm 0.13$ & $7.40 \pm 0.69$ \\
\hline 8 & $4.39 \pm 0.17$ & $96.59 \pm 29.21$ & $21.99 \pm 8.77$ & $0.75 \pm 0.14$ & $6.51 \pm 1.07$ \\
\hline 9 & $4.35 \pm 0.18$ & $94.34 \pm 28.11$ & $23.05 \pm 9.14$ & $0.64 \pm 0.21$ & $6.28 \pm 0.90$ \\
\hline 10 & $4.37 \pm 0.17$ & $96.49 \pm 26.87$ & $18.88 \pm 6.83$ & $0.77 \pm 0.12$ & $6.36 \pm 0.74$ \\
\hline
\end{tabular}

Notes: *Significant differences from baseline $(P<0.05)$. LMM with individual random effects and autoregressive correlation structure: predicted $=$ intercept + time coef + time $^{2}$ coef $_{2}+$ time $^{3}$ coef $_{3}$, where coef $=$ slope of predicted value per I-minute increment of time; coef $f_{2}=$ additive change of predicted value in dependence on squared time $\left(\right.$ minute $\left.^{2}\right) ; \operatorname{coef}_{3}=$ additive change of predicted value in dependence on cubed time (minute ${ }^{3}$ ); *** no quadratic time trend obvious by data assessment; $* * *$ no cubic time trend obvious by data assessment.

Abbreviations: SD, standard deviation; D, luminal diameter; psBFV, peak systolic blood flow velocity; edBFV, end-diastolic blood flow velocity; RI, resistance index; VFave, average volumetric flow; LMM, linear mixed model. 
Table 4 Clinical hemodynamic parameters recorded after the first alfaxalone bolus injection (mean \pm SD)

\begin{tabular}{|c|c|c|c|c|c|c|}
\hline $\begin{array}{l}\text { Time } \\
\text { (minutes) }\end{array}$ & $\begin{array}{l}\text { HR } \\
\text { (bpm) }\end{array}$ & $\begin{array}{l}\text { MAP } \\
(\mathrm{mmHg})\end{array}$ & $\begin{array}{l}\text { SVRI } \\
(\text { dyn*s*cm) }\end{array}$ & $\begin{array}{l}\text { TDCO } \\
\text { (L/minute) }\end{array}$ & $\begin{array}{l}\text { PCCO } \\
\text { (L/minute) }\end{array}$ & $\begin{array}{l}\text { SV } \\
(\mathrm{mL})\end{array}$ \\
\hline \multicolumn{7}{|l|}{ LMM } \\
\hline Intercept & 102.369 & 86.843 & 1258.916 & 5.469 & 5.449 & 51.657 \\
\hline Time $\times$ coef $_{1}$ & 0.191 & 3.136 & 117.183 & -0.068 & 0.028 & 0.267 \\
\hline $\mathrm{Time}^{2} \times \mathrm{coef}_{2}$ & $* *$ & -0.634 & -26.562 & $* *$ & $* *$ & $* *$ \\
\hline $\mathrm{Time}^{3} \times \mathrm{Coef}_{3}$ & $* * *$ & $0.035^{*}$ & $1.490 *$ & $* * *$ & $* * *$ & $* * *$ \\
\hline Baseline & $103 \pm 20$ & $87 \pm 11$ & $1246 \pm 358$ & $5.44 \pm 1.26$ & $5.54 \pm 1.12$ & $53.17 \pm 4.07$ \\
\hline 0.25 & $107 \pm 2 \mid$ & $74 \pm 10^{*}$ & $894 \pm 212^{*}$ & $6.56 \pm 0.64$ & $5.36 \pm 1.33$ & $50.83 \pm 6.37$ \\
\hline I & $104 \pm 22$ & $90 \pm 14$ & $1388 \pm 513$ & $5.18 \pm 1.48$ & $5.34 \pm 1.39$ & $50.67 \pm 5.43$ \\
\hline 2 & $103 \pm 21$ & $91 \pm 14$ & $|4| 2 \pm 457^{*}$ & $5.20 \pm 1.30$ & $5.23 \pm 1.38$ & $49.83 \pm 3.19$ \\
\hline 3 & $100 \pm 25$ & $91 \pm 13$ & $1456 \pm 528^{*}$ & & $5.20 \pm 1.52$ & $49.50 \pm 4.42$ \\
\hline 4 & $104 \pm 21$ & $89 \pm 16$ & $1359 \pm 404 *$ & $5.5 \mathrm{I} \pm 1.36$ & $5.43 \pm 1.48$ & $52.00 \pm 2.74$ \\
\hline 5 & $99 \pm 23$ & $91 \pm 13^{*}$ & $|35| \pm 423 *$ & & $5.39 \pm 1.42$ & $52.17 \pm 5.56$ \\
\hline 6 & $103 \pm 20$ & $90 \pm 12$ & $1320 \pm 380 *$ & $5.29 \pm 1.01$ & $5.44 \pm 1.23$ & $52.00 \pm 2.97$ \\
\hline 7 & $100 \pm 22$ & $90 \pm 12$ & $1315 \pm 377$ & & $5.49 \pm 1.18$ & $53.00 \pm 2.97$ \\
\hline 8 & $104 \pm 19$ & $89 \pm 12$ & $1269 \pm 390$ & $5.31 \pm 1.16$ & $5.58 \pm 1.21$ & $53.50 \pm 3.78$ \\
\hline 9 & $101 \pm 22$ & $90 \pm 12$ & $1286 \pm 370$ & & $5.63 \pm 1.18$ & $53.83 \pm 5.19$ \\
\hline 10 & $104 \pm 19$ & $90 \pm 12$ & $1280 \pm 423$ & $4.69 \pm 2.19$ & $5.63 \pm 1.22$ & $53.80 \pm 5.72$ \\
\hline
\end{tabular}

Notes: *Significant differences from baseline $(P<0.05)$. LMM with individual random effects and autoregressive correlation structure, which yielded the following equation: predicted value $=$ intercept $+\left(\right.$ time $\times$ coef $\left._{1}\right)+\left(\right.$ time $^{2} \times$ coef $\left._{2}\right)+\left(\right.$ time $^{3} \times$ coef $\left._{3}\right)$, where coef $f_{1}=$ slope of predicted value per I-minute increment of time; coef ${ }_{2}=$ additive change of predicted value in dependence on squared time (minute $\left.)^{2}\right)$; coef $_{3}=$ additive change of predicted value in dependence on cubed time (minute $\left.{ }^{3}\right) ; *^{*}$ no quadratic time trend obvious by data assessment; ****no cubic time trend obvious by data assessment.

Abbreviations: SD, standard deviation; HR, heart rate; bpm, beats per minute; MAP, mean arterial pressure; SVRI, systemic vascular resistance index; TDCO, thermodilutional cardiac output; PCCO, pulse contour cardiac output; SV, stroke volume; LMM, linear mixed model.

and third bolus injections, an increase was visible but was not significant. PCCO and SV did not change significantly after each bolus injection.

When data were compared at equivalent time points after the first, second, and third bolus injections, no significant differences were observed among the three bolus injections. Values for GEDI, dPmax, $\mathrm{SpO}_{2}, \mathrm{PETCO}_{2}$, blood temperature, and rectal body temperature were recorded but did not change significantly (data not shown).

Table 5 Clinical hemodynamic parameters recorded after the second alfaxalone bolus injection (mean \pm SD)

\begin{tabular}{|c|c|c|c|c|c|c|}
\hline $\begin{array}{l}\text { Time } \\
\text { (minutes) }\end{array}$ & $\begin{array}{l}\text { HR } \\
(\text { bpm) }\end{array}$ & $\begin{array}{l}\text { MAP } \\
(\mathrm{mmHg})\end{array}$ & $\begin{array}{l}\text { SVRI } \\
(\text { dyn*s*cm) }\end{array}$ & $\begin{array}{l}\text { TDCO } \\
\text { (L/minute) }\end{array}$ & $\begin{array}{l}\text { PCCO } \\
\text { (L/minute) }\end{array}$ & $\begin{array}{l}\text { SV } \\
(\mathrm{mL})\end{array}$ \\
\hline \multicolumn{7}{|l|}{ LMM } \\
\hline Intercept & 102.518 & 89.787 & |298.97| & 5.555 & 5.458 & 52.353 \\
\hline Time $\times$ coef $_{1}$ & 0.011 & 2.768 & 68.869 & -0.145 & -0.031 & -0.322 \\
\hline $\mathrm{Time}^{2} \times$ coef $_{2}$ & $* *$ & -0.573 & $-4.984^{*}$ & 0.031 & $* *$ & $* *$ \\
\hline Time $^{3} \times$ coef $_{3}$ & $* * *$ & $0.031 *$ & $* * *$ & $-0.002^{*}$ & $* * *$ & $* * *$ \\
\hline Baseline & $104 \pm 18$ & $91 \pm 13$ & $1314 \pm 416.47$ & $5.54 \pm 1.30$ & $5.52 \pm 1.19$ & $52.83 \pm 4.17$ \\
\hline 0.25 & $108 \pm 17$ & $75 \pm 10^{*}$ & $907 \pm 250$ & $6.16 \pm 1.02 *$ & $5.34 \pm 1.21$ & $51.83 \pm 3.71$ \\
\hline I & $103 \pm 18$ & $92 \pm 15$ & $1362 \pm 455.56$ & $5.47 \pm 1.27$ & $5.44 \pm 1.35$ & $52.00 \pm 4.56$ \\
\hline 2 & $98 \pm 24$ & $93 \pm 15$ & $1366 \pm 456.87$ & $5.42 \pm 1.15$ & $5.49 \pm 1.32$ & $53.00 \pm 4.98$ \\
\hline 3 & $99 \pm 20$ & $94 \pm 15^{*}$ & $1354 \pm 459.64$ & & $5.52 \pm 1.30$ & $53.83 \pm 4.79$ \\
\hline 4 & $102 \pm 17$ & $93 \pm 15$ & $1646 \pm 760.09$ & $5.31 \pm 1.13$ & $4.95 \pm 1.84$ & $47.50 \pm 13.19$ \\
\hline 5 & $100 \pm 19$ & $91 \pm 14$ & $1588 \pm 70 \mid .75$ & & $4.99 \pm 1.76$ & $48.00 \pm 12.35$ \\
\hline 6 & $103 \pm 16$ & $92 \pm 15$ & $1529 \pm 612.59$ & $5.46 \pm 0.97$ & $5.08 \pm 1.65$ & $48.83 \pm 11.37$ \\
\hline 7 & $102 \pm 20$ & $92 \pm 17$ & $1394 \pm 602.31$ & & $5.29 \pm 1.74$ & $50.20 \pm 12.60$ \\
\hline 8 & $103 \pm 15$ & $90 \pm 13$ & $|472 \pm 580.2|$ & $5.52 \pm 1.03$ & $5.16 \pm 1.60$ & $49.50 \pm I I .4 I$ \\
\hline 9 & $100 \pm 18$ & $90 \pm 12$ & $1466 \pm 574.70$ & & $5.19 \pm 1.58$ & $49.83 \pm 11.20$ \\
\hline 10 & $103 \pm 15$ & $91 \pm 12$ & $1484 \pm 584.62$ & $5.49 \pm 1.21$ & $5.13 \pm 1.55$ & $49.33 \pm 10.93$ \\
\hline
\end{tabular}

Notes: *Significant differences from baseline $(P<0.05)$. LMM with individual random effects and autoregressive correlation structure, which yielded the following equation: predicted value $=$ intercept $+\left(\right.$ time $\times$ coef $\left._{1}\right)+\left(\right.$ time $^{2} \times$ coef $\left._{2}\right)+\left(\right.$ time $^{3} \times$ coef $\left._{3}\right)$, where coef $=$ slope of predicted value per one minute increment of time; coef $f_{2}=$ additive

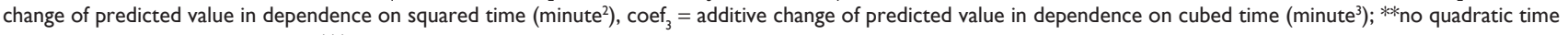
trend obvious by data assessment; ***no cubic time trend obvious by data assessment.

Abbreviations: SD, standard deviation; HR, heart rate; bpm, beats per minute; MAP, mean arterial pressure; SVRI, systemic vascular resistance index; TDCO, thermodilutional cardiac output; PCCO, pulse contour cardiac output; SV, stroke volume; LMM, linear mixed model. 
Table 6 Clinical hemodynamic parameters recorded after the third alfaxalone bolus injection (mean \pm SD)

\begin{tabular}{|c|c|c|c|c|c|c|}
\hline Time (minutes) & HR (bpm) & MAP $(\mathrm{mmHg})$ & SVRI (dyn*s*cm) & TDCO (L/minute) & PCCO (L/minute) & SV $(\mathrm{mL})$ \\
\hline \multicolumn{7}{|l|}{ LMM } \\
\hline Intercept & 98.439 & 91.141 & | 304.977 & 5.485 & 5.550 & 54.180 \\
\hline Time $\times$ coef $_{1}$ & 1.259 & 0.669 & $23.542^{*}$ & 0.015 & -0.035 & -0.364 \\
\hline Time $^{2} \times$ coef $_{2}$ & -0.524 & $* *$ & $* *$ & $* *$ & $* *$ & $* *$ \\
\hline $\mathrm{Time}^{3} \times \mathrm{Coef}_{3}$ & $0.040 *$ & $* * *$ & $* * *$ & $* * *$ & $* * *$ & $* * *$ \\
\hline Baseline & $101 \pm 14$ & $92 \pm 13$ & $1310 \pm 409$ & $5.55 \pm 1.02$ & $5.59 \pm 1.13$ & $54.67 \pm 6.7 \mid$ \\
\hline 0.25 & $105 \pm 14^{*}$ & $78 \pm 12$ & $956 \pm 265^{*}$ & $6.19 \pm 0.89$ & $5.52 \pm 1.11$ & $54.17 \pm 6.37$ \\
\hline I & $102 \pm 14$ & $92 \pm 13$ & $1318 \pm 409$ & $5.57 \pm 1.15$ & $5.60 \pm 1.12$ & $54.50 \pm 5.43$ \\
\hline 2 & $102 \pm 14$ & $93 \pm 13$ & $1343 \pm 409$ & $5.32 \pm 1.09$ & $5.51 \pm 1.08$ & $53.83 \pm 5.19$ \\
\hline 3 & $93 \pm 11$ & $92 \pm 13$ & $1326 \pm 413$ & & $5.51 \pm 1.09$ & $54.00 \pm 5.14$ \\
\hline 4 & $101 \pm 13$ & $93 \pm 13$ & $|34| \pm 4||$ & $5.54 \pm 1.07$ & $5.51 \pm 1.14$ & $53.83 \pm 5.46$ \\
\hline 5 & $93 \pm 11$ & $94 \pm 14$ & $1511 \pm 642$ & & $5.24 \pm 1.50$ & $51.00 \pm 10.92$ \\
\hline 6 & $100 \pm 13$ & $96 \pm 17$ & $1738 \pm 949$ & $5.58 \pm 0.99$ & $4.97 \pm 1.81$ & $48.50 \pm 14.15$ \\
\hline 7 & $93 \pm 10$ & $96 \pm 17$ & $1604 \pm 796$ & & $5.16 \pm 1.57$ & $50.67 \pm 12.06$ \\
\hline 8 & $100 \pm 13$ & $97 \pm 18$ & $1460 \pm 706$ & $5.49 \pm 1.06$ & $4.77 \pm 2.03$ & $46.50 \pm 16.74$ \\
\hline 9 & $92 \pm 10$ & $97 \pm 17$ & $160 \mid \pm 732$ & & $5.11 \pm|.5|$ & $50.17 \pm 10.78$ \\
\hline 10 & $100 \pm 12$ & $97 \pm 17$ & $1509 \pm 537$ & $5.57 \pm 0.92$ & $5.24 \pm 1.22$ & $51.50 \pm 7.23$ \\
\hline
\end{tabular}

Notes: *Significant differences from baseline $(P<0.05)$. LMM with individual random effects and autoregressive correlation structure, which yielded the following equation: predicted value $=$ intercept $+\left(\right.$ time $\times$ coef $\left._{1}\right)+\left(\right.$ time $^{2} \times$ coef $\left._{2}\right)+\left(\right.$ time $^{3} \times$ coef $\left._{3}\right)$, where coef $f_{1}=$ slope of predicted value per I-minute increment of time; coef ${ }_{2}=$ additive change of predicted value in dependence on squared time $\left(\right.$ minute $\left.^{2}\right)$, coef $_{3}=$ additive change of predicted value in dependence on cubed time (minute $\left.{ }^{3}\right) ;{ }^{* *}$ no quadratic time trend obvious by data assessment; *** no cubic time trend obvious by data assessment.

Abbreviations: SD, standard deviation; HR, heart rate; bpm, beats per minute; MAP, mean arterial pressure; SVRI, systemic vascular resistance index; TDCO, thermodilutional cardiac output; PCCO, pulse contour cardiac output; SV, stroke volume; LMM, linear mixed model.

\section{Discussion}

Alfaxalone is a synthetic water-insoluble steroid anesthetic. It has already been used in humans, dogs, and cats in formulations combined with alfadolone using Cremophor ${ }^{\circledR} \mathrm{EL}$ as an excipient. Because Cremophor ${ }^{\circledR}$ EL produced histamine release with often intense allergic reactions, the use of Cremophor ${ }^{\circledR}$ EL was stopped. A new excipient for alfaxalone was found with 2-hydroxypropyl-beta cyclodextrin. This formulation is registered for the use in dogs and cats, but it has also been used in studies on rabbits, sheep, and pigs..$^{5-10,18-20}$

Whereas the study by Keates ${ }^{10}$ previously investigated the induction dose rate of alfaxalone needed for intubation tolerance in pigs, our study investigated the acute short-term hemodynamic and cardiovascular effects of alfaxalone bolus injections in pigs using the PiCCO technique as well as Doppler ultrasonography.

The PiCCO technique uses two different methods of analysis to record, for example, HR, MAP, PCCO, TDCO, SV, and SVRI: pulse contour analysis and transcardiopulmonal thermodilution. The PiCCO technique has been used in many studies and is a well-accepted method in the evaluation of cardiovascular hemodynamic and volumetric parameters. ${ }^{14-17}$

Furthermore, Doppler ultrasound determines vascular diameters, ${ }^{22,23}$ as well as changes in blood flow (psBFV, edBFV, mdBFV, and $\left.\mathrm{V}_{\text {mean }}\right)^{24}$ at the CCA as a noninvasive, accurate method. Volumetric flow can be calculated using these parameters. The CCA is an elastic-type artery much like the truncus brachiocephalicus and the aorta. ${ }^{25,26}$ It can easily be examined by percutaneous ultrasonography, which has already been studied for its suitability to investigate vascular effects of drugs in several studies using goats and rabbits..$^{27-31}$

In our study, ultrasonography of the CCA was always performed by the same person (CB) to avoid variations in the technique of examination. The audible and visible signals as well as the Doppler angle of $45^{\circ}$ to $60^{\circ}$ were always comparable among the single measurements. Consequently, using these two methods, the short-term hemodynamic and volumetric effects of alfaxalone bolus injections in pigs could be investigated in detail.

In our current study, a significant decrease in diameter at the CCA was obvious after every bolus injection at a time point of 15 seconds. The luminal diameter decreased by $3.2 \%$ after the first bolus, 3.9\% after the second bolus, and 4.6\% after the third bolus compared with baseline values, and the diameter increased again to baseline values at a time point of 1 minute. This transient decrease at the vessel could be caused by a direct vasoconstrictoric effect of alfaxalone or a reaction induced by the acute reduction in MAP, as measured at this acute time point.

In this very acute phase, psBFV increased significantly after every bolus injection according to the decrease in 
vessel diameter. Corresponding to this trend, the increase in diameter to normal values at a time point of 1 minute was associated with a decrease in psBFV to baseline values. The averaged volumetric flow in the CCA decreased only slightly in this acute phase (not significantly).

According to the decrease in diameter at the ACC and the increase in psBFV, edBFV decreased (not significantly) and RI increased (not significantly) at 15 seconds after bolus injection.

In contrast to these findings, SVRI decreased significantly at 15 seconds after each bolus, showing a decrease in resistance of the peripheral vessels, possibly caused by vasodilation. Muir et $\mathrm{al}^{32}$ also reported a decrease in SVR after bolus injection of alfaxalone in overdose in cats, explaining their findings with a possible decrease in SV and negative inotropic effects as well as vasodilation. In our study, SV did not change significantly (slightly decreased); consequently, a direct negative inotropic effect of alfaxalone may not be the cause for a decrease in SVRI. Vasodilation has been an often-reported adverse effect of anesthetics such as alfaxalone, which modulate the gammaaminobutyric acid receptors. ${ }^{6,32}$ Ambros et al ${ }^{6}$ reported a decrease of SVR when alfaxalone was used for induction and maintenance of anesthesia in dogs. Since in Ambros et al's study CFI and SV index remained unchanged during the experiment, the authors ruled out a negative inotropic effect of alfaxalone, and explained the decrease in SVR with possible vasodilation. ${ }^{6}$

The already-mentioned decrease in MAP was significant after the first and second bolus injections of alfaxalone at a time point of 15 seconds. After this rapid decrease, MAP immediately started to increase again (not significantly) to reach baseline values at a time point of 1 minute. This reaction could have been caused by a direct vasodilatory effect of alfaxalone at the peripheral vessels. Muir et al ${ }^{32}$ also reported a significant decrease in MAP in cats after a clinical dose of alfaxalone at minute 15 after bolus administration. Directly after bolus injection, a decrease in MAP was visible but was not significant. When they used supraclinical doses, this effect was significant directly after the bolus injection for 30 minutes. As HR and $\mathrm{CO}$ decreased too, Muir et $\mathrm{al}^{32}$ explained their results with a possible direct negative inotropic effect of alfaxalone.

Muir et $\mathrm{al}^{9}$ also conducted a study with dogs, recording no significant changes in MAP when clinical doses were used. Using three or ten times the recommended dose of alfaxalone, these authors observed that MAP significantly decreased at 5 minutes and 1 minute, respectively. After this decrease, MAP remained decreased for 30 minutes and 60 minutes, respectively. Muir et $\mathrm{al}^{9}$ assumed that this hypotension could be caused by peripheral vasodilation and a decrease in cardiac contractile force.

In our study, we used clinical doses of alfaxalone, but the decrease in MAP only lasted for 1 minute. This finding indicates a well-preserved hemodynamic reserve.

Whittem et $\mathrm{al}^{5}$ conducted a study with alfaxalone in cats and administered multiple intravenous bolus injections at clinical doses. At 5 minutes after induction with an alfaxalone bolus injection, MAP decreased but HR decreased (not significantly). After this transient decrease, values for MAP and HR remained stable or began to increase to baseline values again, even when four bolus injections at maintenance doses were administered. These changes were stated to be in acceptable ranges, with only a minor adverse effect of alfaxalone anesthesia.

Ambros et $\mathrm{al}^{6}$ studied the effects of alfaxalone as a continuous infusion in dogs. They found a decrease in MAP at a time point of 5 minutes after induction of anesthesia, whereas HR increased at this time point. They explained these findings with a reflectory increase in HR because of hypotension. As the values of $\mathrm{CFI}$ and $\mathrm{SV}$ index remained stable during anesthesia, Ambros et $\mathrm{al}^{6}$ stated that alfaxalone has no or only limited negative inotropic effects.

At the same time point as MAP decreased, HR increased transiently and was clinically obvious after every bolus injection in our current study, but this finding was significant only after the third bolus injection. This increase could be a reflectory reaction to the decreased MAP. In a study conducted with sheep, ${ }^{20} \mathrm{HR}$ increased immediately after administration of alfaxalone and returned to baseline values after 20 minutes, whereas arterial blood pressure remained unchanged. Bösing et $\mathrm{al}^{33}$ found similar results in their study in cats: after administration of alfaxalone as a bolus injection, HR increased and lasted up to 10 minutes. Both studies evaluated the increase in HR as a minor adverse effect of alfaxalone.

Muir et $\mathrm{al}^{9}$ studied the effect of clinical and supraclinical doses of alfaxalone in dogs. They also reported an increase in HR, but only at supraclinical doses of alfaxalone bolus injections (three times and ten times higher). Muir et $\mathrm{al}^{9}$ also noticed that MAP and SVR decreased, but HR increased, and the authors assumed that vasodilation of peripheral vessels had caused these effects.

In contrast to their abovementioned findings, Muir et $\mathrm{al}^{32}$ in 2009 reported decreased HRs at supraclinical doses of alfaxalone in cats. In their later study, they noted that HR significantly decreased at 1 minute after bolus administration, when the dose was ten times higher than the clinical dose, 
and at 5 minutes after bolus administration, when the dose was three times higher than the clinical dose. HR remained decreased until 30 minutes. In clinical doses, there was no significant decrease in HR. Therefore, one might conclude that the decrease in HR depends on the dose of alfaxalone. Muir et $\mathrm{al}^{32}$ explained their findings with direct cardiac depression and vasodilation and stated that more detailed investigation is necessary to arrive at this assumption.

In our study, a clinical dose of alfaxalone was used. As HR and TDCO increased but MAP decreased and SV remained unchanged, we assumed no direct cardiac depression caused by alfaxalone when used in clinical doses (as this would be indicated by a decrease in HR, TDCO, MAP and SV). Also, PCCO and SV did not change significantly.

TDCO transiently increased after every bolus injection at a time point of 15 seconds, but this trend was only significant after the second bolus injection. $\mathrm{CO}$ is calculated via the formula

$$
\mathrm{HR} \times \mathrm{SV}
$$

Regarding the figures, $\mathrm{CO}$ increased because HR increased and $\mathrm{SV}$ remained unchanged. Muir et $\mathrm{al}^{9}$ reported increased TDCO when using three times the recommended dose of alfaxalone and a decrease in $\mathrm{CO}$ when using ten times the recommended dose of alfaxalone in dogs. At both dosages, HR increased at these time points, whereas SVR slightly decreased and MAP decreased. Muir et $\mathrm{al}^{9}$ concluded that $\mathrm{CO}$ reacts in this way because of peripheral vasodilation and a negative inotropic effect of alfaxalone.

In a study conducted with cats receiving supraclinical doses of alfaxalone bolus injections, ${ }^{32} \mathrm{CO}$ decreased at a time point of 1 minute at a dose three times higher than the recommended dose of alfaxalone and at a time point of 5 minutes at a dose ten times higher than the recommended dose. Muir et al $^{32}$ suggested a decrease in SV and negative inotropic effects may have caused the decrease in CO.

When comparing the recorded data after each of the three bolus injections, we saw no cumulative effect either statistically or clinically. The elimination half-life of alfaxalone in dogs is approximately 24 minutes, ${ }^{8}$ but to our knowledge, the elimination half-life of alfaxalone in pigs has not yet been described. As we did not see a cumulative effect, we assume the half-life in pigs to be longer, but we cannot rule out a cumulative effect. Table 7 shows an overview during the cited study protocols.

\section{Study limitations}

Limitations of the study could be seen. Multiple post hoc Student's $t$-tests for paired samples were used to assess differences between the value for each time point during the 10-minute time frame after the alfaxalone bolus injection and the baseline value. Adjustment of $P$-values for the number of comparisons made would have increased the likelihood of a type 2 error, and a larger sample size would have been necessary to yield sufficient power for a detailed analysis because the probability of detecting false significant differences (differences by chance) increases with the number of tests performed. Because of that, in our study we used nonadjusted $P$-values as statistical measures of importance and considered our results explorative.

To rule out any influence by the saline bolus injections, we tested the effect of saline bolus injections with PiCCO and vascular sonography without an alfaxalone bolus injection. The saline bolus injection did not influence any parameter measured.

Table 7 Cited studies in comparison

\begin{tabular}{|c|c|c|c|c|}
\hline Author & Species & Study protocol & Alfaxalone dose & Results \\
\hline Muir et al ${ }^{32}$ & Cat & Bolus injection & clinical dose: $5 \mathrm{mg} / \mathrm{kg}$ & Decrease of SVR; decrease of MAP in minute 15 \\
\hline Muir et al ${ }^{32}$ & Cat & Bolus injection & $\begin{array}{l}\text { Supraclinical dose: } 15 \mathrm{mg} / \mathrm{kg} \\
\text { (three times higher) }\end{array}$ & $\begin{array}{l}\text { Decrease of MAP minute } 0-30 \text {; decrease of } \mathrm{HR} \\
\text { in minute } 5-30 \text {; decrease of } \mathrm{CO} \text { in minute I }\end{array}$ \\
\hline Muir et $\mathrm{al}^{32}$ & Cat & Bolus injection & $\begin{array}{l}\text { Supraclinical dose: } 50 \mathrm{mg} / \mathrm{kg} \\
\text { (ten times higher) }\end{array}$ & $\begin{array}{l}\text { Decrease of MAP minute } 0-30 \text {; decrease of } \mathrm{HR} \text { in } \\
\text { minute } 1-30 \text {; decrease of } \mathrm{CO} \text { in minute } 5\end{array}$ \\
\hline Ambros et $\mathrm{al}^{6}$ & Dog & $\begin{array}{l}\text { Induction, } \\
\text { maintenance (CRI) }\end{array}$ & $\begin{array}{l}\text { Clinical dose: } 2 \mathrm{mg} / \mathrm{kg} \text { induction, } \\
0.07 \mathrm{mg} / \mathrm{kg} / \mathrm{min} \mathrm{CRI}\end{array}$ & $\begin{array}{l}\text { Decrease of SVR; decrease of MAP in minute } 5 \text {; } \\
\text { increase of HR in minute } 5\end{array}$ \\
\hline Muir et al ${ }^{9}$ & Dog & Bolus injection & $\begin{array}{l}\text { Supraclinical dose: } 6 \mathrm{mg} / \mathrm{kg} \\
\text { (three times higher) }\end{array}$ & $\begin{array}{l}\text { Decrease of MAP in minute } 5-30 \text {; increase of HR; } \\
\text { increase of TDCO }\end{array}$ \\
\hline Muir et al9 & Dog & Bolus injection & $\begin{array}{l}\text { Supraclinical dose: } 20 \mathrm{mg} / \mathrm{kg} \\
\text { (ten times higher) }\end{array}$ & $\begin{array}{l}\text { Decrease of MAP in minute I-60; increase of HR; } \\
\text { decrease of TDCO }\end{array}$ \\
\hline Whittem et al ${ }^{5,9}$ & Cat & $\begin{array}{l}\text { Single and multiple } \\
\text { bolus injections }\end{array}$ & Clinical dose: $5 \mathrm{mg} / \mathrm{kg}$ & $\begin{array}{l}\text { Decrease of MAP in minute } 5 \text { after bolus I; } \\
\text { decrease of HR in minute } 5 \text { after bolus I }\end{array}$ \\
\hline Andaluz et $\mathrm{a}^{20}$ & Sheep & Bolus injection & Clinical dose: $2 \mathrm{mg} / \mathrm{kg}$ & Increase of $\mathrm{HR}$ minute $0-20$ \\
\hline
\end{tabular}

Abbreviations: SVR, systemic vascular resistance; MAP, mean arterial blood pressure; HR, heart rate; CO, cardiac output; CRI, constant rate infusion; TDCO, thermodilutional cardiac output. 
The bolus injections of alfaxalone led to intense reactions of the vessels in the first 60 seconds. Because of these reactions, hemodynamic measurements by the thermodilution method were technically possible at the earliest at 1 minute after bolus injection. In between, the pulse contour analysismeasured values for SVRI, CO, MAP, and HR were taken for analysis of data. A study performed by Gruenewald et $\mathrm{al}^{34}$ demonstrated that intra-abdominal hypertension affects the precise measurement of PCCO. Piehl et a ${ }^{35}$ concluded that rapid changes in blood pressure or intravascular volume lead to imprecise values of PCCO. Both of these studies recorded accurate $\mathrm{PiCCO}$ values after recalibration with thermodilution.

\section{Conclusion}

Alfaxalone bolus injections caused a transient significant decrease in vessel diameter at the CCA after each bolus injection at a time point of 15 seconds.

Simultaneously, HR, psBFV, and TDCO significantly increased, whereas MAP and SVRI significantly decreased.

Vascular diameter reincreased toward baseline data after these acute changes, and psBFV significantly decreased; however MAP and SVRI significantly increased until the time point of 10 minutes after bolus injection.

Bolus injections of alfaxalone produced biphasic vascular and hemodynamic changes in pigs. Indeed, these alterations were only associated with transient changes in $\mathrm{CO}$ and did not lead to significant changes of volumetric flow, indicating a well preserved function of the cardiovascular system. No cumulative effects were clinically and statistically evident when comparing the data among the three bolus injections.

Although the direct intense vascular and hemodynamic effects of an alfaxalone bolus were quickly compensated in young pigs, this bolus may be carefully used in patients with ventricular dysfunction.

\section{Disclosure}

The authors report no conflicts of interest in this work.

\section{References}

1. Child KJ, Currie JP, Dis B, Dodds MG, Pearce DR, Twissell DJ. The pharmacological properties in animals of CT1341 - a new steroid anaesthetic agent. Br J Anaesth. 1971;43(1):2-13.

2. Dodman NH. Complications of saffan anaesthesia in cats. Vet Rec. 1980;107(21):481-483.

3. Prys-Roberts C, Sear J. Steroid anaesthesia. Br J Anaesth. 1980;52(4): 363-365.

4. Brewster ME, Estes KS, Bodor N. Development of a non-surfactant formulation for alfaxalone through the use of chemically-modified cyclodextrins. J Parenter Sci Technol. 1989;43(6):262-265.
5. Whittem T, Pasloske KS, Heit MC, Ranasinghe MG. The pharmacokinetics and pharmacodynamics of alfaxalone in cats after single and multiple intravenous administration of Alfaxan at clinical and supraclinical doses. J Vet Pharmacol Ther. 2008;31(6):571-579.

6. Ambros B, Duke-Novakovski T, Pasloske KS. Comparison of the anesthetic efficacy and cardiopulmonary effects of continuous rate infusions of alfaxalone-2-hydroxypropyl-beta-cyclodextrin and propofol in dogs. Am J Vet Res. 2008;69(11):1391-1398.

7. Psatha E, Alibhai HI, Jimenez-Lozano A, Armitage-Chan E, Brodbelt DC. Clinical efficacy and cardiorespiratory effects of alfaxalone, or diazepam/fentanyl for induction of anaesthesia in dogs that are a poor anaesthetic risk. Vet Anaesth Analg. 2011;38(1):24-36.

8. Ferré PJ, Pasloske K, Whittem T, Ranasinghe MG, Li Q, Lefebvre HP. Plasma pharmacokinetics of alfaxalone in dogs after an intravenous bolus of Alfaxan-CD RTU. Vet Anaesth Analg. 2006;33(4):229-236.

9. Muir W, Lerche P, Wiese A, Nelson L, Pasloske K, Whittem T. Cardiorespiratory and anesthetic effects of clinical and supraclinical doses of alfaxalone in dogs. Vet Anaesth Analg. 2008;35(6):451-462.

10. Keates $\mathrm{H}$. Induction of anaesthesia in pigs using a new alphaxalone formulation. Vet Rec. 2003;153(20):627-628.

11. Zaki S, Ticehurst K, Miyaki Y. Clinical evaluation of Alfaxan$\mathrm{CD}(\mathrm{R})$ as an intravenous anaesthetic in young cats. Aust Vet $J$. 2009;87(3):82-87.

12. Taboada FM, Murison PJ. Induction of anaesthesia with alfaxalone or propofol before isoflurane maintenance in cats. Vet Rec. 2010;167(3):85-89.

13. Keates H, Whittem T. Effect of intravenous dose escalation with alfaxalone and propofol on occurrence of apnoea in the dog. Res Vet Sci. 2012;93(2):904-906.

14. Hüeter L, Schwarzkopf K, Preussler NP, et al. Measuring cardiac output in one-lung ventilation: a comparison of pulmonary artery and transpulmonary aortic measurements in pigs. $J$ Cardiothorac Vasc Anesth. 2004;18(2):190-193.

15. Rupérez M, López-Herce J, García C, Sánchez C, García E, Vigil D. Comparison between cardiac output measured by the pulmonary arterial thermodilution technique and that measured by the femoral arterial thermodilution technique in a pediatric animal model. Pediatr Cardiol. 2004;25(2):119-123.

16. López-Herce J, Rupérez M, Sánchez C, García C, García E. Correlation between cardiac output measured by the femoral arterial thermodilution technique pulmonary arterial and that measured by contour pulse analysis in a paediatric animal model. J Clin Monit Comput. 2006;20(1):19-23.

17. Bajorat J, Hofmockel R, Vagts DA, et al. Comparison of invasive and less-invasive techniques of cardiac output measurement under different haemodynamic conditions in a pig model. Eur J Anaesthesiol. 2006;23(1):23-30.

18. Roan R. Use of alfaxalone in rabbits. Vet Rec. 2009;164(6):188.

19. Grint NJ, Smith HE, Senior JM. Clinical evaluation of alfaxalone in cyclodextrin for the induction of anaesthesia in rabbits. Vet Rec. 2008;163(13):395-396.

20. Andaluz A, Felez-Ocaña N, Santos L, Fresno L, García F. The effects on cardio-respiratory and acid-base variables of the anaesthetic alfaxalone in a 2-hydroxypropyl- $\beta$-cyclodextrin (HPCD) formulation in sheep. Vet J. 2012;191(3):389-392.

21. Stiegler H, Klews PM. Hämodynamische grundlage [Basic hemodynamics]. In: Kubale R, Stiegler H, editors. Farbkodierte Duplexsonographie. Stuttgart, Germany: Thieme; 2002:93. German.

22. Mueck-Weymann M, Wupperman T. Physiologie und pathophysiologie des blutflusses [Physiology and pathophysiology of blood flow]. In: Wupperman T, editor. Sonographie der Gefaesse. Munich, Germany: Urban and Fischer; 1999:21-27. German.

23. Lin GS, Spratt RS. Hemodynamic imaging with pulsatility index and resistive-index color Doppler US. Radiology. 1997;204(3):870-873.

24. Widder B, Goertler M. Doppler- und Duplexsonographie der Hirnversorgenden Arterien. Heidelberg, Germany: Springer-Verlag; 2004:21. German. 
25. Carroll BA. Carotid sonography. Radiology. 1991;178(2):303-313.

26. Liebich H-G. Funktionelle Histologie der Haussäugetiere und Vögel. Stuttgart, Germany: Schattauer; 2010:135-136. German.

27. Lee SW, Hankes GH, Purohit RC, et al. Comparative study of ultrasonography and arteriography of the carotid artery of xylazine-sedated and halothane-anesthetized goats. Am J Vet Res. 1990;51(1):109-113.

28. Baumgartner CM, Koenighaus H, Ebner JK, Henke J, Schuster T, Erhardt WD. Cardiovascular effects of fentanyl and propofol on hemodynamic function in rabbits. Am J Vet Res. 2009;70(3):409-417.

29. Baumgartner CM, Koenighaus H, Ebner JK, Henke J, Schuster T, Erhardt WD. Cardiovascular effects of dipyrone and propofol on hemodynamic function in rabbits. Am J Vet Res. 2009;70(11):1407-1415.

30. Baumgartner C, Bollerhey M, Ebner J, Laacke-Singer L, Schuster T, Erhardt W. Effects of ketamine-xylazine intravenous bolus injection on cardiovascular function in rabbits. Can J Vet Res. 2010;74(3):200-208.

31. Baumgartner C, Bollerhey M, Ebner J, Schuster T, Henke J, Erhardt W. Effects of medetomidine-midazolam-fentanyl IV bolus injections and its reversal by specific antagonists on cardiovascular function in rabbits. Can J Vet Res. 2010;74(4):286-298.
32. Muir W, Lerche P, Wiese A, Nelson L, Pasloske K, Whittem T. The cardiorespiratory and anesthetic effects of clinical and supraclinical doses of alfaxalone in cats. Vet Anaesth Analg. 2009;36(1):42-54.

33. Bösing B, Tünsmeyer J, Mischke R, Beyerbach M, Kästner SB. Clinical usability and practicability of Alfaxalone for short-term anaesthesia in the cat after premedication with Buprenorphine. Tierarztl Prax Ausg K Kleintiere Heimtiere. 2012;40(1):17-25. German [with English abstract].

34. Gruenewald M, Renner J, Meybohm P, Höcker J, Scholz J, Bein B. Reliability of continuous cardiac output measurement during intraabdominal hypertension relies on repeated calibrations: an experimental animal study. Crit Care. 2008;12(5):R132.

35. Piehl MD, Manning JE, McCurdy SL, et al. Pulse contour cardiac output analysis in a piglet model of severe hemorrhagic shock. Crit Care Med. 2008;36(4):1189-1195.
Open Access Animal Physiology

\section{Publish your work in this journal}

Open Access Animal Physiology is an international, peer-reviewed, open access journal publishing original research, reports, reviews and commentaries on all areas of animal physiology. The manuscript management system is completely online and includes a very

\section{Dovepress}

quick and fair peer-review system. Visit http://www.dovepress.com/ testimonials.php to read real quotes from published authors. 